\title{
“ESPACE, LIEU, PAYSAGE” : LA REPRÉSENTATION DU PAYSAGE DANS LES DÉCORS ITALIENS DE LA RENAISSANCE
}

\section{Denis Ribouillault}

Éditions Ligeia | « Ligeia »

2007/1 N²3-76 | pages 33 à 45

ISSN 0989-6023

Article disponible en ligne à l'adresse :

https://www.cairn.info/revue-ligeia-2007-1-page-33.htm

Distribution électronique Cairn.info pour Éditions Ligeia.

(C) Éditions Ligeia. Tous droits réservés pour tous pays.

La reproduction ou représentation de cet article, notamment par photocopie, n'est autorisée que dans les limites des conditions générales d'utilisation du site ou, le cas échéant, des conditions générales de la licence souscrite par votre établissement. Toute autre reproduction ou représentation, en tout ou partie, sous quelque forme et de quelque manière que ce soit, est interdite sauf accord préalable et écrit de l'éditeur, en dehors des cas prévus par la législation en vigueur en France. Il est précisé que son stockage dans une base de données est également interdit. 


\title{
"ESPACE, LIEU, PAYSAGE": LA REPRÉSENTATION DU PAYSAGE DANS LES DÉCORS ITALIENS DE LA RENAISSANCE
}

\author{
Denis Ribouillault
}

Plusieurs essais récents sur la peinture de paysage ont signalé de nouvelles directions de recherche et, tout particulièrement, l'intérêt qu'il y aurait à analyser la notion de "paysage" en rapport avec les notions de "lieu" et d' "espace". Il s'agirait d'une part de repenser le paysage comme une image du lieu et, d'autre part, de réfléchir à la manière dont le paysage réel et le paysage dans ses représentations sont appréhendés spatialement. L'exercice requiert de dépasser les distinctions traditionnellement établies en histoire de l'art entre les différents media artistiques - peinture, architecture, sculpture, paysage / espace réel -, pour engager une réflexion sur la relation du spectateur à l'espace construit et informé par ces media.

Dans la préface de la seconde édition de Landscape and Power, publiée en 2002, W. J. T. Mitchell suggère une telle orientation. Que la représentation du " paysage " possède une signification politique et idéologique, thèse soutenue dans l'ouvrage publié pour la première fois en 1994, est un fait qui, selon l'auteur, semble avoir été largement accepté par la communauté des chercheurs. Il propose donc de donner un nouvel intitulé à son ouvrage, Space, place, and landscape, c'est-à-dire "Espace, Lieu, et Paysage"'. Un paysage, écrit-il, est un espace, la "vue", la représentation d'un lieu. À la fois dans la tradition phénoménologique et dans la tradition marxiste, c'est-à-dire tant chez Gaston Bachelard ou Martin Heidegger que chez Henri Lefebvre, Michel de Certeau et Michel Foucault, l'espace et le lieu sont les termes essentiels de l'analyse. Le "paysage" n'intervient qu'en tant que "concept d'encadrement esthétique des propriétés véritables de l'espace et du lieu" (aesthe- tic framing of the real properties of space and places) $)^{2}$. Les trois termes doivent donc être considérés comme une triade plus ou moins inséparable : si le "lieu" (place) est une localité, un emplacement, un site précis, l' "espace" (space) est un lieu dans lequel s'exerce une pratique - c'est "l'espace social" dont parle Henri Lefebvre dans son ouvrage classique La Production de l'espace, ouvrage dont les implications dans le domaine de l'histoire de l'art sont fondamentales ${ }^{3}$. Le "paysage" (landscape) est ce site, entendu à travers le medium de la vision, le sens de la vue, comme image ou "vue". Scape / scope en anglais traduit d'ailleurs précisément l'importance de la médiation du regard, in visu, dans la construction d'un "paysage". En français, il donne "scopique" ou les suffixes "scope" ou "scopie", sont liés au sens de la vue. En termes de mouvement pourtant, scope en anglais signifie aussi espace, envergure.

Dans son ouvrage, Representing Place. Landscape Painting and Maps, le philosophe Edward S. Casey propose lui aussi une approche qui

\section{NOTES}

J'ai bénéficié pour l'élaboration et la rédaction de cet article des conseils et indications de Hervé Brunon, Amold Witte et Jérémie Koering que je tiens à remercier.

1. W. J. T. Mitchell, "Preface to the Second Edition of Landscape and Power ; Space, Place, and Landscape", in Landscape and Power, W. J. T. Mitchell (éd.), The university of Chicago Press, Chicago-London, 2002, p. vii-xii.

2. Ibidem, p. viii.

3. Henri Lefebvre, La Production de l'espace, Anthropos, Paris, 2000 (1971). 
serait centrée non pas tant sur les contingences historiques et sociales des images que sur leurs modes de perceptions visuelles et physiques : "Je suis moins concerné, explique-t-il, par la manière dont la représentation fonctionne, par son contenu culturel, sa position historique ou sa charge politique que par la manière dont la représentation du paysage nous touche, nous les spectateurs, non pas socialement ou politiquement mais du point de vue de l'expérience (experientially) [...] Ce n'est pas seulement une affaire d'“œil et d'esprit" (comme l'a judicieusement exprimé Merleau-Ponty) mais de l'œil - et de la main, du pied, du dos, du cou..."4.

Il est significatif que les travaux d'Edward Casey aient intéressé en premier lieu les historiens étudiant les décors peints de paysages et de cartes dans les palais italiens de la Renaissance. Francesca Fiorani, dans sa récente étude sur les décors cartographiques dans l'Italie de la Renaissance et Cristina Bragaglia Venuti, dans un article sur les paysages topographiques de la Terza Loggia au Vatican soulignent l'importance des concepts de lieu et d'espace pour leurs analyses. 5 . Fiorani explique :

"Tandis que les philosophes modernes à partir d'Emmanuel Kant ont étudié la notion d'espace comme une entité abstraite et incorporelle et l'ont confronté à celle de temps, Casey se concentre sur les relations entre lieu et espace. Comme l'espace, le lieu est une des conditions de notre être-aumonde mais, à l'inverse de l'espace, il conserve certains éléments de matérialité et fait référence à un€ localisation représentée avec ses caractéristiques uniques. En ce sens, le lieu diffère également du site, qui est "le lieu réduit à sa localisation et à sa position dans l'espace". Un site est donc un lieu qui est représenté par le moyen d'une grille et privé de ses caractéristiques individuelles. Les notions de lieu et de site, que Casey lui-même applique à la peinture de paysage et aux cartes en général, sont essentielles pour expliquer le langage des cartes de la Renaissance : dans ces images, un site n'est pas seulement situé mathématiquement sur une grille cartographique mais aussi décrit avec une grande abondance de détails qualitatifs".6.

Pour F. Fiorani, comme pour C. Bragaglia Venuti, ces termes offrent donc une possibilité supplémentaire de conceptualisation et d'analyse du langage cartographique et topographique, mais la relation spatiale entre cartes et paysages peints et l'espace qui les contient, c'est-à-dire l'espace de la galerie ou de la salle et, au-delà, le paysage réel, n'est considérée que de manière relativement superficielle? ${ }^{7}$. Leurs enquêtes, aussi précieuses soientelles, se concentrent sur la production de l'image, de sa forme et son iconographie, plutôt que sur sa réception et sa perception dans l'espace ${ }^{8}$.

L'étude de la représentation du paysage dans les villas et les palais italiens de la Renaissance offre une possibilité intéressante de renouveler cette approche. Le paysage prend, à partir de la fin du $X V^{e}$ siècle et tout au long du $X V I^{e}$ siècle, une place sans cesse plus importante dans les décors peints. On le trouve souvent représenté dans les frises ou dans de petits tableaux insérés parmi les décors de grotesques, mais il peut également recouvrir entièrement les parois d'une pièce. Les sujets peuvent être mythologiques, religieux, topographiques ou

\section{NOTES}

4. Edward S. Casey, Representing place: landscape painting and maps, University of Minnesota Press, Minneapolis, 2002, p. xvi. 5. Francesca Fiorani, The marvel of maps : art, cartography and politics in Renaissance Italy, Yale University Press, New-HavenLondon, 2005, p. 5 ; Cristina Bragaglia Venuti, "Étienne Dupérac e i paesaggi della Loggia di Pio IV", Rivista dell'Istituto Nazionale d'Archeologia e Storia dell'Arte, 25, n` 57, 2002 (2003), p. 279-309.

6. Fiorani, op. cit., p. 5.

7. Ibidem, p. 8-9.

8. C'est également le cas des travaux de Svetlana Alpers sur les rapports entre cartographie et peinture dans l'art hollandais du XVII' siècle. Voir Svetlana Alpers, The art of describin : Dutch art in the seventeenth century, Murray, London, 1983 (trad. fr. : L'Art de dépeindre, la peinture hollandaise au XVIIe siècle, Flammarion, Paris, 1995). Plus qu'aux historiens de l'art, c'est aux historiens des jardins et aux géographes que l'on doit un véritable renouvellement des méthodologies concernant l'analyse de la perception du paysage. Pour la contribution des géographes à une réflexion sur la notion d'espace et de lieu voir, parmi une ample bibliographie, Yi-Fu Tuan, Space and Place: The Perspective of Experience, Mineapolis University Press, Mineapolis, 1977 ; Augustin Berque, Être humains sur la terre. Principes d'étique de l'écoumène, Paris, 1996 ; Jean-Marc Besse, Voir la terre. Six essais sur le paysage et la géographie, Arles-Versailles, 2002, p. 115-146. Sur le mouvement dans les jardins et le paysage voir Landscape Design and the Experience of Motion, Michel Conan (éd.), Dumbarton Oaks Research Library and Collection, Washington D.C., 2003 et Malgorzata Szafranska, "Place, time and movement: a new look at Renaissance gardens", in Studies in the History of Gardens and Designed Landscapes, 26, 3, July-Sept. 2006, p. 194-208. Sur la notion de jardin comme « lieu », voir John Dixon Hunt, Greater Perfections : The Practice of Garden Theory, London, 2000, p. 1 ; Philippe Nys, Le jardin exploré. Une herméneutique du lieu, Besançon, 1999, vol. 1, p. 77-79. 
simplement inventés - on parle alors de "paysages de fantaisie". L'importance et le développement du genre à la Renaissance sont le reflet d'un sentiment renouvelé pour la représentation de la Nature, qui dérive pour l'essentiel de la littérature classique, des Géorgiques de Virgile au De Vita Solitaria de Pétrarque. Selon Vitruve (De Arch., 7, 5, 2) et Pline (Hist. Nat., 35, 116-117), la peinture de paysage était également largement utilisée par les Romains pour orner leurs villas, indications qui déterminèrent de nombreux peintres à la Renaissance à s'essayer dans ce genre nouveau. De l'essai fondateur de Jacob Burckhardt sur le sentiment de la Nature à la Renaissance, aux écrits d'Ernst Gombrich et de Richard Turner sur la peinture de paysage en Italie, cette inspiration classique des décors a été amplement soulignée par les historiens de l'art'.

Sans dénier l'importance de ces sources, nous voudrions suggérer ici une nouvelle approche, une manière de considérer le paysage dans les décors qui n'a pas été envisagée par les historiens de l'art, à savoir la relation entre le paysage peint et le paysage réel ou, plus précisément, entre l'espace virtuel construit par le paysage peint sur les murs à l'intérieur du palais et l'espace ou "lieu" constitué en paysage à l'extérieur de celui-ci. Afin de souligner l'interdépendance entre ces deux types de paysages à la Renaissance, nous limiterons nos analyses aux décors topographiques, à la représentation de lieux réels. En effet, la manière dont les cycles de paysages topographiques ont généralement été conçus à l'intérieur de l'espace physique des palais a souvent été calculée avec une grande attention de manière à créer un dialogue tant visuel que symbolique avec le paysage urbain ou rural visible au dehors. Plutôt que de s'attacher à la représentation du "lieu" dans l'espace pictural bidimensionnel de la représentation, nous analyserons son inscription dans l'espace tridimensionnel du palais et du paysage réel ${ }^{10}$.

\section{La ville et la campagne}

Ce dialogue entre intérieur et extérieur, déjà essentiel dans la peinture romaine, est clairement perceptible dans l'un des premiers cycles topographiques italiens: les fresques du Bon et $d u$ Mauvais Gouvernement peintes par Ambrogio Lorenzetti dans la Sala dei Nove du Palazzo Publico de Sienne vers 1338-1340 (fig. 1)". Les sujets étaient organisés sur les murs en rapport étroit avec le paysage réel à l'extérieur du palais. La représentation des effets du Bon et du Mauvais
Fig. 1. Ambrogio Lorenzetti, Sala dei Nove, parois est et nord, fresque, vers 1338-1340, Sienne, Palazzo Pubblico.

Gouvernement sur la campagne, respectivement sur les murs est et ouest, occupe la moitié sud de la salle, qui ouvre, à travers l'unique fenêtre sur la Piazza del Mercato, où les produits du contado étaient vendus et, au-delà, sur le panorama de la campagne siennoise ${ }^{12}$. La partie nord de la salle

9. Voir Jacob Burckhardt, La Civilisation de la Renaissance en Italie, Paris, 1958, chap. IV ; Emst H. Gombrich, "The Renaissance Theory of Art and the Rise of Landscape", in Norm and Form: Studies in the Art of the Renaissance, Phaidon, London, 1966, p. 107-121, trad. fr. A. Lévêque, "La théorie artistique de la Renaissance et l'essor du paysage", in L'Écologie des images, Flammarion, Paris, 1983, p. 15-43 ; Richard A. Turner, The Vision of Landscape in Renaissance Italy, Princeton, Princeton University Press, 1966. Sur les décors de paysages, on verra aussi la synthèse de Eva Börsch-Supan, Garten, Landschaft und Paradiesmotive im Innenraum : Eine ikonographische Untersuchung, Bruno Hessling, Berlin, 1967.

10. En histoire de l'art, on considère généralement l'espace pictural à l'intérieur des limites du cadre et plus rarement l'espace réel dans lequel le spectateur évolue. Parmi les études désormais classiques, on citera par exemple John White, The birth and rebirth of pictorial space, London, Faber \& Faber, 1957 (Naissance et Renaissance de l'espace pictural, trad. C. Fraixe, Adam Biro, Paris, 1992).

11. On notera que certains historiens de l'art ont émis l'hypothèse que les décors de paysages et de natures mortes antiques ont pu avoir une influence sur les fresques du Bon et Mauvais gouvernement. Voir Charles Sterling, La Nature morte de l'Antiquité à nos jours, Éd. des Musées Nationaux, Paris, 1952, p.19-21, 37 38.

12. Le point de vue théorique et symbolique des fresques serait donc logiquement la salle elle-même. John White suggérait à juste titre que la diminution d'échelle observée dans la fresque du contado siennois sur la paroi est tendait à reproduire une vision de l'espace qui eût été celle d'une personne contemplant le territoire depuis le sommet de la porte de la ville, mais il situe le point focal de la composition au centre de la cité. Voir White. op. cit., 1957, p. 93-95. On retiendra aussi l'hypothèse de Uta Feldges qui met le panorama peint en relation avec les vues réelles depuis le palais. Selon elle, il s'agit d'une vue prise depuis la tour du Palazzo Publico. Cf. Uta Feldges, Landschaft als topographisches Porträt. Die wieder beginnt der europäischen Landschaftmalerei in Siena, Bentelli, Bern, 1980, p. 64. 
(parois est et ouest), orientée vers la ville, est, au contraire, dédiée à la représentation de l'espace urbain et des effets opposés de la Tyrannie et de la Justice sur la ville. La relation entre l'espace fictif des représentations picturales et l'espace réel était également remarquable sur la paroi nord. En effet, il est probable que les magistrats de l'Assemblée des Neufs siégeaient en-dessous de la fresque figurant les vertus du Bon Gouvernement. Pour un visiteur, le parallèle visuel entre les magistrats et les vertus peintes qu'ils incamaient et, pour ainsi dire, "redoublaient", devait paraître évident ${ }^{13}$. Ces échos visuels, entre l'espace virtuel de la peinture et l'espace réel - espace social où s'exerçaient des relations politisées -, ancraient les représentations hautement symboliques dans la réalité visuelle et soulignaient leur virtualité tangible.

À Rome, ce dialogue fit son apparition à la fin du $X V^{e}$ siècle avec la décoration des premières villas construites à la périphérie de la ville. Inspirés par les modèles et la littérature classiques, leur architecture et leurs décors s'inscrivaient au sein de la dialectique bien connue entre ville et campagne qui qualifie toute la littérature pastorale et les traités d'agronomie, de Juvénal et Virgile jusqu'à Sannazzaro et Agostino Gallo. Suivant le conseil de Vitruve, Alberti avait recommandé de situer la villa sur une hauteur, afin d'en magnifier la grandeur et d'offrir des vues sur la campagne environnante, et suffisamment près de la ville pour que le maître puisse s'y rendre rapidement si ses affaires l'exigeaient ${ }^{14}$. La grande majorité des résidences suburbaines italiennes, la Villa Médicis, la Villa Lante, la Villa Montalto à Rome, le Château Saint-Ange, ou encore la Villa du Belvédère à Florence articulaient leurs vues vers la ville d'un côté, vers la campagne de l'autre ${ }^{15}$.

La Villa du Belvédère à Rome, construite comme retraite pour le pape Innocent VIII à la fin $\mathrm{du} \mathrm{XV}^{\mathrm{e}}$ siècle, obéissait pleinement à cette règle. Selon Vasari, Pinturicchio peignit dans la loggia nord des vues de Rome, Milan, Gênes, Florence, Venise et Naples (1487-1489) ${ }^{16}$. Les vues de villes étaient situées sur le mur sud, en direction de la ville, et contrastaient avec le splendide panorama de la campagne romaine qui ouvrait au nord, avec le Monte Mario et le Ponte Milvio.

Obéissant aux recommandations de Vitruve dans le livre VII de son De Architectura, le peintre a divisé la surface des murs au moyen de pilastres et arches peints qui reprennent le dessin de la loggia opposée. Des descriptions du XVIII ${ }^{e}$ siècle mentionnent également des balustrades peintes entre les pilastres qui auraient accentué encore l'illusionnisme de l'ensemble ${ }^{17}$. Deux autres loggias existaient au nord, de part et d'autre de la loggia principale. Selon Chattard, elles étaient décorées de paysages et de scènes de chasse. Ces fresques campagnardes devaient évoquer l'importance des prati comme zone de chasse pour la cour papale et contraster, là encore, avec les scènes urbaines représentées dans la loggia principale. Cette opposition entre ville et campagne dans les sujets peints reflétait la situation réelle de la villa par rapport à son environnement : au sud, la façade dominait Rome, tandis qu'au nord, elle ouvrait sur la campagne.

L'importance de la situation géographique de la villa suburbaine entre ville et campagne est également rendue explicite dans la décoration de la Villa d'Agostino Chigi située à Rome, sur les rives du Tibre. Le poète Blosio Palladio avait déjà célébré le site dans sa description de la villa en 1512 (Suburbanum Augustini Chisii), elle-même inspirée par le poème de Martial sur la villa antique de Julius Martialis située non loin de là sur le Janicule (IV, 64) : la villa était "située au milieu de la ville", si bien que l'on voyait d'un côté les maisons, de l'autre la campagne.

Vers 1515-1516, Baldassare Peruzzi devait prendre en compte cette spécificité de la villa dans la décoration de la Sala delle Prospettive, une vaste salle au premier étage transformée fictivement en une loggia ouverte sur l'extérieur (fig. 2). Les vues peintes entre les colonnes correspondaient clairement aux vues réelles depuis la villa. Dans la partie est de la salle, c'est-à-dire vers Rome, le peintre a représenté, dans un paysage urbain, des monuments bien reconnaissables comme l'Hôpital de Santo

13. Randolph Stam, Ambrogio Lorenzetti. Le palais communal, Sienne, trad. J. F. Piquet, Hazan, Paris, 1995, p. 30.

14. Alberti, De Re Aed., 5, 2.

15. Claude Mignot, "Les loggias de la Villa Médicis à Rome", Revue de l'art, 19, 1973, p. 50-61, p. 56.

16. Vasari, Le Vite, G. Milanesi (dir.), G. C. Sansoni, Florence, 1880, vol. III, p. 458.

17. David R. Coffin, "The Self-Image of the Roman Villa during the Renaissance", Architectura. Zeitschrift für Geschichte der Baukunst, 28/2, 1998, p. 181-203, p. 182-183. 
Spirito ou la Tour des Milices. Dans la partie ouest de la salle, les paysages évoquent, à l'inverse, la campagne qui s'étendait dans cette direction au $X V I^{e}$ siècle, avec ses fermes, ses champs et ses petits villages ${ }^{18}$.

Certains décors peints de paysages portèrent cette identité entre extérieur et intérieur à un niveau encore supérieur. Les peintres ne se contentaient pas seulement d'imiter ou d'évoquer le paysage réel visible à l'extérieur ni de situer la villa au sein de son environnement, mais dessinaient une véritable carte du territoire à l'échelle tridimensionnelle de la salle.

Évoluer à l'intérieur d'une salle décorée de paysages se présentait ainsi comme l'équivalent d'une promenade véritable dans ce paysage, tout comme parcourir un jardin était souvent la métaphore d'un voyage sur le territoire : un parcours physique et non plus simplement mental entre les différents lieux représentés, parcours linéaire dans une galerie ou une loggia, circulaire et périégétique dans une salle de plan rectangulaire. La projection mentale du spectateur, dans l'espace de l'image à deux dimensions, était désormais complétée, en trois dimensions, par les mouvements physiques de celui-ci à travers la galerie ou la salle ${ }^{19}$. L'expérience visuelle des peintures se rapprochait ainsi de l'expérience réelle d'un paysage qui, par définition, ne peut être saisi en un seul coup d'œil. Le paysage est, dans son essence, ce que Jean-Paul Sartre appelle une "totalité détotalisée", quelque chose qui, tout en étant appréhendé comme un tout, n'est cependant pas réductible à la somme de ses parties. Le paysage est donc toujours partiellement "invisible", ou limité à sa visibilité littérale. Il englobe physiquement le spectateur et par conséquent interdit toute possibilité d'être saisi visuellement dans sa totalité. Edward Casey forge, pour réfléchir sur cette question, le concept d'unité "panperceptuelle" : "En tant qu'unité pan-perceptuelle, le paysage défie la simple imitation, c'est-à-dire, tout effort de le réduire à une sorte d'objet défini (ou à un groupe d'objets) qui peut être appréhendé en une seule fois, et par conséquent en une seule image" ${ }^{\prime 20}$. Dans une salle, le regard se pose sur les paysages peints comme il se pose sur certains éléments d'un paysage dans la réalité ; en somme, un regard flottant dont le comportement imprévisible est déterminé par les caprices et le caractère unique du sujet regardant. Nous sommes ici à l'opposé d'un regard albertien où le paysage serait vu comme
Fig. 2. Baldassare Peruzzi, Sala delle Prospettive, fresque, vers 1516-1517, Rome, Villa Farnesina.

l'accessoire d'un champ de vision ajusté à l'œil de l'observateur, comme un décor de théâtre limité symboliquement par les quatre côtés de la fenêtre ${ }^{21}$.

En faisant se "pénétrer réciproquement" le paysage et le palais ou la villa, la salle peinte s'entendait comme un véritable microcosme territorial, comme le centre propulseur du territoire luimême $^{22}$. Son décor signalait le pouvoir fédérateur

\section{NOTES}

18. Sur la Villa Farnesina, voir Christoph L. Frommel, La Villa Farnesina a Roma, Franco Panini Editore, Modena, 2003, p. 126-133.

19. Le paysage est ici entendu comme la juxtaposition d'éléments de ce paysage, disparates, choisis et regroupés arbitrairement dans un seul espace. La saisie successive des différents éléments du paysage de la part du spectateur est l'équivalent au niveau de la perception visuelle d'une expérience physique dans ce paysage. En d'autres termes, les mouvements du corps à travers l'espace, la marche, sont réduits au doigt dessinant un parcours à la surface de l'image ou au mouvement des yeux sur cette image. Explorer l'image, c'est transformer les mouvements véritables du doigt ou des yeux en un mouvement mental du corps tout entier. Sur la perception du paysage et les rapports entre "promenade" et "regard", voir les remarques fondamentales de Daniel Arasse, Le Détail. Pour une histoire rapprochée de la peinture, Flammarion, Paris, 1992, p. 163-166.

20. Casey, op. cit., p. 6. Sartre développe la notion de "totalité détotalisée" dans L'Étre et le Néant. Essai d'ontologie phénoménologique, Gallimard, Paris, 1943.

21. Alberti, De Pict., I, 12 et 19.

22. J'emprunte l'expression à Pierre Grimal qui caractérise ainsi une tendance fondamentale de l'architecture romaine vis-à-vis du paysage et des jardins. Voir Pierre Grimal, Les jardins romains, PUF, Paris, 1969 (2`éd.), p. 244. 
du Seigneur, dont l'omniscience visuelle et politique était matérialisée sur les parois et offerte aux regards des visiteurs et des hôtes.

$\mathrm{La}$ galerie des cartes géographiques au Vatican, conçue pour le pape Grégoire XIII vers 1580 , est un des décors les plus célèbres qui illustre ce "travestissement géographique" de l'espace architectural grâce aux décors peints. Dans la Galerie des cartes, les scènes du plafond figurent des épisodes fondateurs de l'histoire des saints qui se sont déroulés dans les territoires représentés sur les parois dans les cartes sous-jacentes. En marchant le long de la galerie, le visiteur effectuait, dès lors, un véritable pèlerinage imaginaire dans les lieux sacrés de la péninsule italienne. En effet, la galerie toute entière avait été conçue comme une image de l'Italie. Elle matérialisait, dans sa longueur, la chaîne montagneuse des Apennins de part et d'autre de laquelle s'étendaient les diverses provinces figurées par les cartes : en marchant du nord au sud, on pouvait voir, sur la paroi ouest, les provinces situées vers la mer Méditerranée et, à l'est, celles qui bordent l'Adriatique. La galerie était d'ailleurs désignée par le nom d'Ambulatio ou en italien spasseggio, qui signifie déambulation, promenade. Elle introduisait ainsi une dimension narrative, "la linéarité vectorielle d'un voyage" avec son enchaînement rigoureux d'étapes, recréant l'expérience symbolique du pèlerinage. Dans une célèbre description anonyme, le regard omniscient du pape, matérialisé par les cartes, est comparé à celui de Jupiter observant les hommes du haut de la voie lactée $e^{23}$.

L'exemple le plus ancien de cette spatialisation cardinale des décors de paysages et de cartes est, à ma connaissance, un décor daté de la première moitié du XVe siècle (1417-1432) au Palazzo della Masseria à Mantoue. Le palais était le siège de la Masseria, ferme générale qui gérait les entrées financières et les dépenses de la commune et du contado. Les fresques, qui se développaient de manière ininterrompue sur les quatre lunettes et parois de la salle rectangulaire, constituent la plus ancienne représentation de la ville de Mantoue et de son territoire. La ville est représentée de manière très précise ainsi que les routes, fleuves, lacs et châteaux qui formaient la ceinture territoriale défensive de l'État des Gonzague. La dimension cartographique du décor est assez évidente car les portes de la ville ou les différents châteaux sont désignés par des inscriptions en lettres gothiques. La paroi la mieux conservée au sud figure justement la partie sud du territoire. Il est donc légitime de supposer que cette orientation géographique correcte des cartes-paysages se poursuivait sur les trois autres parois $^{24}$. Cette idée d'un espace pictural unitaire, développé sur l'ensemble des parois d'une salle, existait déjà à Mantoue dans les fresques de Pisanello pour la Corte Vecchia du Palais Ducal ${ }^{25}$.

Ce décor pourrait très bien avoir influencé la décoration de la bien plus célèbre Camera d'Oro du château de Torchiara près de Parme qui présente, en effet, de grandes similitudes avec ce décor mantouan (fig. 3). Pier Maria Rossi, seigneur de Torchiara, fit décorer, à la fin des années 1450, une des salles de son château avec toutes les forteresses qui constituaient son petit État. Ce décor, d'une puissante originalité, allie la célébration de sa carrière de condottiere à celle de la dame de ses pensées, Bianca Pellegrini, pour qui le château avait été conçu et édifié. Représentée sur chaque voûtain, à une échelle démesurée par rapport au paysage, Bianca effectue, en quelque sorte, un parcours vir-

\section{NOTES}

23. Voir à propos de la perception des cartes dans les cycles italiens de la Renaissance les remarques de Christian Jacob : Christian Jacob, L'Empire des cartes. Approche théorique de la cartographie à travers l'histoire, Albin Michel, Paris, 1992, p. 126-129. Rolando Ferri, "Una passeggiata in Italia. L'anonima Ambulatio gregoriana", in Lucio Gambi, Antonio Pinelli (dir.), La Galleria delle Carte Geografiche in Vaticano. The Gallery of Maps in the Vatican, Mirabilia Italiae, Franco Cosimo Panini, Modena, 1994, p. 73-81. Un autre décor, moins célèbre, décoré de vues de villes orientées géographiquement se trouve au Palazzo della Loggia à Bagnaia, près de Viterbe. Voir Denis Ribouillault, "Il cardinale Gambara e il Palazzo della Loggia a Bagnaia", in Villa Lante a Bagnaia, actes du colloque international (Viterbo, 2004), Sabine Frommel (dir.), Electa, Milano, 2005, p. 44-53.

24. Voir Ilaria Toesca, "Un antica vedute di Mantova e del suo territorio", in Mantova gonzaghesca nelle stampe e nelle monete, catalogue d'exposition (Mantova, 1981), Comune di Mantova, Assessorato alla cultura, Mantova, 1982, p. 9-16, p. 10-12 ; Gianna Suitner, in Mina Gregori (éd.), Pittura a Mantova dal Romanico al Settecento, Milano, 1989, p. 215-217 ; E. Marani, "La Masseria di Mantova e i suoi affreschi", in $\mathrm{La}$ Masseria a Mantova. Città e castelli alla fine del Medioevo, Mantova, 1983, p. 11-45.

25. Sur la perception des fresques de Pisanello et leur continuum narratif, voir les remarques de Joanna Wood-Marsden, The Gonzaga of Mantua and Pisanello's Arthurian Frescoes, Princeton University Press, 1988, Princeton, p. 95-96. 
tuel dans l'espace cartographique ${ }^{26}$. Comme à Mantoue, la disposition des différentes places fortes sur les quatre portions de la voûte d'arête du plafond et dans les lunettes correspond à une volonté de réalisme topographique. Sans doute aidés par une carte de la région ou peut-être du propre État de Rossi, le peintre et les exécutants ont défini un point, au centre du territoire, à partir duquel toute la représentation s'articule. Les châteaux situés au nord de ce point comme Castel Maria, Sant'Andrea, Castrignano ou Rivalta furent peints sur la voûte et la lunette nord, tandis que ceux à l'est, au sud, et à l'ouest de ce point, prirent place respectivement dans les voûtains et les lunettes est, sud et ouest ${ }^{27}$. L'omniscience visuelle sur le territoire qu'offrait à son seigneur ce fascinant décor était complétée et pour ainsi dire "redoublée" par les vues depuis son palais puisque la salle ouvre directement sur une des loggias grâce à une paroi percée à cet effet. Pour le visiteur, le rapport entre peintures et paysage est immédiatement saisissable. Sabatino degli Arienti note ainsi à propos de la salle : "extra thalamum specula est ex qua omnis Aemilia et Liguria speculantur" $"$.8.

Dans le Latium, le décor un peu plus tardif de la loggia du Palais Farnèse de Gradoli, situé au cœur du territoire farnésien sur les rives du lac de Bolsena, au nord de Rome, présente aussi de telles caractéristiques. D'un point de vue typologique, il renvoie directement aux fresques de la Sala delle Prospettive à la Famésine. Il fut exécuté selon toute vraisemblance entre 1521 et 1524 . La loggia qui ouvrait au nord et à l'est sur un magnifique paysage était conçue à nouveau comme un véritable belvédère. En effet, les parois sud et ouest étaient décorées d'une architecture illusionniste qui complétait l'organisation architectonique des parois opposées, et à travers laquelle on pouvait admirer des paysages peints. Malgré leur mauvais état de conservation, ces paysages sont toujours lisibles. Les scènes représentées, l'Enlèvement de Ganymède ou Diane et Actéon, ont été copiées à partir de gravures contemporaines ${ }^{29}$. Seuls les paysages diffèrent des modèles originaux. Aux profils de villes nordiques qui occupent l'arrière-plan des gravures, le peintre de Gradoli a substitué des vues typiques de la campagne romaine qui font ainsi écho au paysage réel visible depuis le palais. Dans le fond du paysage avec Diane et Actéon, la forteresse rappelle étroitement celle de Bolsena et le village dans Jupiter et Ganimède correspond à la position de Marta sur les rives du lac. De même, on peut reconnaître, dans un autre paysage, le motif d'un temple hexagonal, une allusion très probable à celui que construisit Antonio da Sangallo il Giovane, l'architecte du palais, sur l'île Bisentina, au centre du lac de Bolsena, où fut enterré l'ancêtre de la famille Ranuccio Famese. La loggia, avec ses vues et ses paysages peints, offrait donc au spectateur une vision véritablement panoramique du paysage. Maria Luisa Polidori note d'ailleurs que les paysages des parois peintes sont correctement orientés en fonction de la géographie du territoire. Ainsi, sur la paroi sud vers Bolsena, des vues de Bolsena sont reconnaissables ; de même à l'ouest pour Capodimonte, "comme pour confondre les paysages réels et visibles avec les paysages peints, eux aussi toutefois réellement existants et qu'il aurait été possible d'admirer si les parois n'avaient pas existé" 30 .

26. La fresque a été attribuée à Benedetto Bembo (v 1415 - v 1493) et Bonifacio Bembo (? - après 1460). Chacune des forteresses représentées avait été conquise par la force au cours d'une période où l'hégémonie milanaise sur la région s'était brusquement affaiblie, à la suite de la mort en 1447 du duc de Milan, Filippo Maria Visconti. En 1449, la possession des territoires usurpés fut rendue légitime et ratifiée sous l'autorité de Francesco Sforza, à qui Rossi s'était intelligemment rallié, reconnaissant dans la personne du grand condottiere le futur duc de Milan. La représentation des châteaux dans la Camera peregrina aurea peut donc être vue comme une affirmation picturale des prétentions de Rossi sur les territoires conquis. Voir Joanna Woods-Marsden, "Pictorial legitimation of Territorial Gains in Emilia : The iconography of the "camera peregrina aurea" in the Castle of Torchiara", in Renaissance Essays in Honor of Craig Hugh Smyth, A. Morrogh et F. Superbi Gioffredi (éd.), Giunti Barbera, Firenze, 1985, vol. 2, p. 553-568.

27. Sur l'orientation des vues sur la voûte et la dimension cartographique du décor, voir Luciano Summer, "Considerazioni topographiche sugli affreschi della Camera d'Oro a Torchiara », Parma nell'arte, 11, 1979, p. 51-61.

28. Voir Angelo Marino, "Logge e altane come belvedere : dai castelli emiliani ai palazzi romani”, in Il castello : origini, realtà, fantasia, Paolo Portoghesi (dir.), Corbo, Ferrara, 1985, p. 112 136, p. 113.

29. Sur la décoration de la loggia, voir Flaminia Gennari Santori, "Il Palazzo Famese di Gradoli", in Flaminia Gennari Santori, Sabina Robert, Francesca Vicarelli, Decorazioni farnesiane nei palazzi di Valentano, Capodimonte e Gradoli, Produzione artistica e committenza nel territorio romano - secolo XVI, Claudia Cieri Via (dir.), De Luca, Roma, 1996, p. 131-158, en particulier p. 141-146.

30. Voir Maria Luisa Polidori, "ll Palazzo Famese di Gradoli", in Palazzi Baronali del Lazio, Renato Lefevre (dir.), Lunario Romano, 20, 1991, gruppo culturale di Roma e del Lazio, Fratelli Palombi editori, Roma, 1990, p. 275-284, p. 283. 
Cette orientation correcte des vues par rapport au territoire, qui accroît l'illusionnisme du décor et la relation à la topographie locale, s'explique par l'importance symbolique du territoire puisque cette région du Latium est la terre d'origine de la puissante dynastie des Farnèse. Paysage mythologique et territoire étaient donc fondus en un seul et même paysage $^{31}$.

L'importance des vues sur le territoire pour la définition sociale des familles aristocratiques, comme signe d'une domination territoriale ancestrale, est particulièrement manif este dans le Latium, où le souvenir des célèbres villas romaines est fortement présent ${ }^{32}$. Comme à Gradoli, les territoires du Latium n'étaient jamais appréhendés indépendamment de leur histoire ancienne et mythique. La superposition de paysages topographiques aux scènes les plus célèbres de la mythologie signalait les nouveaux propriétaires comme les héritiers directs des héros et des dieux qui avaient jadis régné sur le territoire.

À Tivoli, lieu de villégiature des empereurs comme des poètes les plus célèbres de l'époque augustéenne, le paysage et l'histoire sont, encore aujourd'hui, indissociablement liés. Lorsque le cardinal Hippolyte d'Este s'établit dans l'ancienne cité romaine au milieu du $X V^{*}$ siècle, l'histoire mythique du lieu et les sites antiques préexistants disséminés sur le territoire alentour allaient fournir les coordonnées essentielles du programme de sa nouvelle villa et de ses célèbres jardins. Comme l'a démontré David Coffin, le jardin est, en effet, une représentation symbolique et miniaturisée du territoire de Tivoli avec son fleuve Aniene qui se jette dans le Tibre à Rome, puis dans la mer. La Fontaine de Tivoli, adossée à la bourgade au nord-est du jardin, représente la fameuse cascade sur le fleuve Aniene située au nord-est de la ville, tandis que la Fontaine de Rome, correctement orientée en direction de la ville éternelle, contient un modèle de la ville antique avec ses portes et ses monuments les plus célèbres. L'allée des Cent Fontaines qui relie les deux fontaines figure le cours du fleuve Aniene traversant le territoire ${ }^{33}$.

Dans des travaux récents, nous avons pu démontrer que le symbolisme géographique des jardins avait été également appliqué aux paysages peints des parois à l'intérieur de la villa, dans le Salone du rez-de-chaussée qui conduit aux jardins ${ }^{34}$. L'axe Tivoli-Rome a été précisément matérialisé dans l'espace de la pièce (fig. 4). Sur la paroi nord- est, la fontaine, dite Fontanina, évoque, comme la Fontaine de Tivoli dans le jardin, la cascade avec le temple de la Sybille situés au nord-est de la ville. Sur la paroi opposée, la grande vue de la villa est orientée vers le sud-est, en direction de Rome. Le Salone est donc métaphoriquement baigné par les eaux de l'Aniene tout comme le sont concrètement le jardin et le territoire. Débouchant de la petite fontaine rustique à une extrémité de la salle, la rivière réapparaît dans la fresque sur la paroi opposée. $\mathrm{La}$ vasque de la fontaine en forme de barque reposant sur de gros poissons figure d'ailleurs le cours d'eau s'épanchant virtuellement dans l'espace de la pièce.

Cette disposition est complétée par l'orientation correcte d'autres vues topographiques visibles entre les colonnes feintes qui transforment la pièce en une vaste loggia. Les vues des fontaines du jardin sur les parois de la salle, la Fontaine de l'orgue au nord et la Fontaine de Tivoli à l'est, évoquent leur exacte disposition dans l'espace du jardin. Plus intéressantes encore sont les vues nouvellement identifiées des villas antiques de Tivoli, la Villa de Manlius Vopiscus, la Villa d'Horace et la Villa d'Auguste que Pirro Ligorio, l'architecte de la Villa d'Este, avait étudiées avec attention pour son Libro delle antiche ville tiburtine. Elles aussi ont été disposées dans l'espace de la pièce de manière à refléter leur position réelle sur le territoire. Saisies dans leur ensemble, les vues peintes du Salone consti-

31. Le choix d'épisodes mythologiques liés à la chasse s'expliquerait également par le fait que le paysage visible depuis la loggia, était considéré comme un des terrains de chasse privilégiés des Farnèse dans la région. Voir Sofia Varoli Piazza, "Barchi, parchi e giardini farnesiani. Il patrimonio arboreo", I Quadernidi Gradoli, 7-8, 1990, p. 33-50, p. 34-38.

32. Sur l'importance du paysage dans la définition sociale des familles aristocratiques du Latium voir nos travaux récents : Denis Ribouillault, Paysage et Pouvoir. Les décors topographiques à Rome et dans le Latium au XVI' siècle, Thèse de doctorat, Université Paris I Panthéon-Sorbonne, 2006 ; ainsi que Tracy L. Ehrlich, Landscape and identity in early modern Rome : villa culture at Frascati in the Borghese era, Cambridge University Press, Cambridge, 2002.

33. Voir David Coffin, La Villa d'Este at Tivoli, Princeton University Press, Princeton, 1960, p. 78-92.

34. Pour une étude détaillée des paysages du Salone voir Denis Ribouillault, "Le Salone de la Villa d'Este à Tivoli : un théâtre des jardins et du territoire", Studiolo. Revue d'histoire de l'art de l'Académie de France à Rome, 3, 2005, p. 65-94. Voir également Denis Ribouillault, "Paesaggio dipinto, Paesaggio reale. Notes sur une fenêtre de la Villa d'Este à Tivoli", Schifanoia. Rivista dell'Istituto di Studi Rinascimentali di Ferrara, 2006, à paraître. 
tuent donc une représentation véritablement homothétique du territoire de Tivoli et des jardins ${ }^{35}$. Typologiquement et spatialement, la Villa d'Este se trouve située au centre du circuit des anciennes villas de Tibur dont elle emprunte les caractéristiques principales : elle couronnait la colline ouvrant du haut de ses terrasses sur le vaste panorama ; elle abritait un homme illustre, puissant, distingué dans les arts et les lettres comme dans la politique de son temps ; elle apportait, par sa beauté et sa magnificence, la gloire à la région de Tivoli. Le Salone, avec ses paysages, illustrait l'importance de Tivoli comme villégiature des grands de l'Antiquité et faisait du programme de la Villa d'Este un véritable acte de refondation.

$\mathrm{La}$ "spatialisation" des vues sur le paysage s'explique donc à Tivoli, tout comme à Gradoli ou à Torchiara, par la nécessité du propriétaire de la villa de se situer non seulement géographiquement mais aussi culturellement, idéologiquement et historiquement par rapport au paysage territorial ${ }^{36}$. Parce qu'il participe idéologiquement à l'identité du seigneur, l'espace du paysage et les "lieux" qui le constituent sont miniaturisés, modélisés dans l'espace de la salle et reliés entre eux par des réseaux de significations rendus explicites au visiteur. Gaston Bachelard, écrivant dans La poétique de l'Espace, ne percevait d'ailleurs bien l' "espace-paysage" que dans la miniature comme "le géomètre voit exactement la même chose dans deux figures semblables dessinées à des échelles différentes", le principe étant que "je possède d'autant mieux le monde que je suis plus habile à le miniaturiser" ${ }^{37}$. Jean Baudrillard, en commentant l'œuvre du plasticien contemporain Charles Matton, spécialiste des espaces miniatures, souligne, quant à lui, la "puissance magique de l'équivalent miniaturisé des choses, au contraire de leur agrandissement monumental : en les concentrant dans un espace fabuleux, parce que plus petit, on en retrouve la quintessence. Représenter un espace, une scène, en plus petit, c'est nous forcer à y entrer de plus près, beaucoup plus efficacement, c'est une manière de nous initier $[\ldots]]^{\prime 38}$.

Les rapports entre le paysage vu et vécu à l'extérieur du palais et le paysage peint et perçu à l'intérieur de celui-ci ne répondaient pas seulement à des exigences idéologiques mais renvoyaient également à des considérations d'ordre thérapeutique. À la Renaissance, les recommandations de la part des architectes de construire des villas sur des positions élevées par rapport au paysage s'accompagnaient d'une véritable science de l'orientation en fonction des vents et des zones d'ombre et d'ensoleillement. Là encore, les architectes, Alberti le premier, avaient suivi les conseils des Anciens et surtout de Vitruve. Comme l'explique Bettina Bergmann à propos de la villa antique, "les salles romaines devaient être orientées en relation aux quatre coins du monde, aux heures du jour, aux saisons et aux

35. L'homothétie désigne la propriété de deux figures qui sont telles que chacun de leurs points se correspondent deux à deux sur des droites qui passent par un point fixe (le centre d'homothétie). Le rapport des distances du centre d'homothétie à deux points correspondants appelés points homologues est toujours le même.

36. Un autre décor qui mérite d'être mentionné dans le cadre de cette étude est celui de la Sala dei Cinquecento au Palazzo Vecchio de Florence, réalisé vers 1565 par Giorgio Vasari et son atelier. Pour l'Entrée de Jeanne d'Autriche en 1565 on décora les parois de la Sala Grande de grands panneaux représentant dix villes ou places de villes toscanes distribuées dans la salle selon leur orientation réelle par rapport à Florence. Selon l'hypothèse de Loren Partridge, les vues de Sienne et de Pise devaient être placées au centre des parois est et ouest respectivement, de manière à faire écho aux épisodes des guerres de Sienne et de Pise représentées au plafond. Pise aurait été flanquée de gauche à droite par les cités se trouvant au sud-ouest, nord-ouest et nord de Florence (Volterra. Pistoia, Prato et Fiesole) et Sienne (de gauche à droite) par les cités au sud-est (Borgo Sansepolcro, Arezzo, Montelpulciano et Cortona). Au plafond, seize vues de villes, combinant figures allégoriques, inscription et veduta, s'organisent aux deux extrémités de la salle autour de la représentation du quartier de la ville dont elles dépendaient administrativement et juridiquement. Comme l'étaient les vues temporaires sous-jacentes, elles sont correctement orientées et reflètent la réalité géographique du territoire. Le plafond s'apparente ainsi à une carte schématique de l'État toscan ainsi qu'à un diagramme de l'administration cosimienne. Voir Randolph Starn, Loren Partridge, Arts of power : three halls of state in Italy, 1300-1600, University of California Press, Berkeley-Oxford, 1992, p. 176, 348-349 et Philippe Morel, "L'état médicéen au XVI' siècle : de l'allégorie à la cartographie" Mélanges de l'École française de Rome (Italie et méditerranée), 105, 1993, p. 93-131, p. 111-119. 37. Gaston Bachelard, Poétique de l'espace, PUF, Paris, 1957, chap. 7, "La miniature", p. 140-167, p. 140-142.

38. Texte de Jean Baudrillard sur Charles Matton de 1987. Voir Charles Matton, préf. Jean Baudrillard, Hatier, Paris, 1991, p. 204 : "La reconstitution de lieux miniaturisés autorise l'exercice d'un pouvoir inconcevable sur la grandeur nature. Les libertés totales, prises sur l'objet, ont un caractère profanatoire". 
vents" ${ }^{\prime 39}$. Presque toutes les villas de la Renaissance furent construites dans ce souci. La Villa du Belvédère d'Innocent VIII au Vatican, la Villa Medici de Fiesole, la Villa Badoer à Fratta Polesine en Vénétie ou la Villa de Belriguardo près de Ferrare en sont de parfaits exemples ${ }^{40}$. Chaque pièce devait être orientée en fonction de sa destination particulière afin qu'elle soit, ou non, protégée du soleil et des vents. Les décors peints des villas sont un reflet direct de ces conceptions d'ordres thérapeutiques et pratiques. Parmi les thèmes profanes recommandés par Silvio Antoniano au cardinal Pietro Aldobrandini pour la décoration des villas en 1603 , on trouve ainsi, outre la peinture de paysage, les quatre saisons, les quatre éléments, et les heures du jour ${ }^{41}$. Le nom des pièces dans les villas de la Renaissance révèle souvent ce souci d'orientation de la villa par rapport à son environnement. À la Villa Grazioli de Frascati par exemple - mais c'est aussi le cas d'un nombre important de villas -, la Stanza del Giorno ou del Sole se trouve à l'ouest de l'édifice, tandis que celle du Riposo ou della Notte, se trouve située dans l'aile est opposée $e^{42}$. Comme pour les paysages peints mentionnés plus haut, les représentations des vents et des cartes du ciel dans les décors étaient également presque toujours orientées. Dans la Sala Meridiana de la Tour des Vents au Vatican, le vent du nord est représenté sur la paroi nord, le vent sud sur la paroi sud, etc. Au Palais Farnèse de Caprarola, dans la Salle de la Mappemonde, la carte du ciel au plafond est logiquement orientée en rapport avec les points cardinaux ${ }^{43}$.

Dans un passage célèbre du De Re Aedificatoria, Alberti étend la qualité thérapeutique du paysage vu depuis la villa aux paysages peints eux-mêmes, reconnaissant que "Notre âme se réjouit grandement lorsque nous voyons représentés en peinture les beautés des paysages ou des ports, des scènes de pêche, de chasse ou de baignade, des jeux agrestes, des fleurs et des feuillages" et que "contempler des peintures de sources et de ruisseaux fait beaucoup de bien aux fiévreux" ${ }^{\text {. }}$. Pour Alberto Lollio aussi, les vues sur la campagne "ont une telle efficacité que, non seulement quand elles sont véritables et faites des mains de la Nature, mais aussi quand elles sont mortes et feintes par l'art, elles réjouissent les esprits et réconfortent les yeux de manière admirable"45. Utiles pour guérir de la fièvre, elles sont aussi un remède efficace contre la mélancolie. Giovanni Battista Armenini recomman- dait ainsi en 1586 de peindre dans les loggias des "choses joyeuses tels que de splendides paysages". Dans de semblables décors, rien, précise-t-il, ne doit apparaître "qui puisse provoquer la mélancolie ou l'ennui" "46. L'intérêt thérapeutique de la peinture de paysage figurait aussi dans la littérature scientifique. Le philosophe et médecin Girolamo Cardano pensait lui aussi que les peintures et les descriptions littéraires de paysages soulageaient un esprit mélan-

\section{NOTES}

39. Bettina Bergmann, "Exploring the Grove : Pastoral Space on Roman Walls”, in Pastoral Landscape, John Dixon Hunt (éd.), National Gallery of Art, Washington D.C. ; Studies in the History of Art, 36, Hanover-London, 1992, p. 21-46, p. 38.

40. Sur l'orientation des palais de la Renaissance en fonction du climat, des saisons et des vents et l'influence des écrits de Vitruve, on renverra à la récente contribution de Deborah Howard, "Seasonal Apartments in Renaissance Italy", in Artibus et Historiae, 43, 2001, p. 127-135. Sur la Villa du Belvédère, voir David R. Coffin, "Pope Innocent VIII and the Villa Belvedere", in Studies in late medieval and renaissance painting in honor of Millard Meiss, New-York, 1977, p. 88-97. Sur la Villa de Belriguardo, voir les travaux récents de Maria Teresa Sambin De Norcen, "I miti di Belriguardo", in Nuovi antichi : committenti, cantieri, architetti 1400-1600, Richard Schofield (dir.), Electa, Milano, 2004, p. 17-65, qui démontrent la manière dont l'entière conception de la villa est subordonnée aux vues et à l'orientation des vents et du soleil.

41. La lettre a été récemment republiée dans Clare Robertson, "Silvio Antoniano and the painted decoration of the villa Belvedere at Frascati", Römisches Jahrbuch der Bibliotheca Hertziana, vol. 35, 2003-2004, p. 417-430, p. 429-430.

42. Almamaria Tantillo Mignosi (éd), Villa Grazioli a Grottaferrata : concorso per il restauro del giardino, Kappa, Roma, 1993, p. 16.

43. Nicola Courtright, The Papacy and the Art of Reform in Sixteenth Century Rome. Gregory XIII's Tower of the Winds in the Vatican, Cambridge University Press, Cambridge, 2003, p. 72, p. 81-83. Loren Partridge, "The Room of Maps at Caprarola, 1573-75", Art Bulletin, 72, 1995, p. 413-444, fig. 1 et 2.

44. Alberti, De Re Aed., 9, 4, 805-807. Des recherches scientifiques modemes ont d'ailleurs démontré la pertinence de cette valeur thérapeutique de la contemplation du paysage. Voir Roger Ulrich, "View through a window May Influence Recovery from Surgery", Science, 224, 1984, p. 420-421.

45. A . Lollio, "Lettera in Lode della Villa", in Lettere di XIII huomini illustri nella quali sono due libri di diversi altri autori, Venezia, 1564, p. 449 ; cité dans Vincenzo Mancini, Lambert Sustris a Padova : la villa Bigolin a Selvazzano, Biblioteca publica comunale, Sevazzano dentro, 1993, p. 40 (ma traduction). La lettre fut publiée de manière autonome dès 1544 .

46. Giovan Battista Armenini, De' veri precetti della pittura, Francesco Tebaldini, Ravenna, 1596, p. 198. 
colique aussi bien que la nature elle-même ${ }^{47}$. Une personnalité comme Giulio Mancini, à la fois critique d'art et physicien, avait naturellement introduit de telles idées sur les relations entre art et thérapie dans ses Considerazione sulla pittura (1620). Il y discute de l'effet des différents sujets picturaux sur l'humeur des spectateurs ${ }^{48}$.

Les salles-paysages de la Renaissance, conçues en fonction des coordonnées fondamentales du paysage environnant, avec leurs formes quadrangulaires évoquant la perfection de la Création, rythmées par la représentation des saisons, fonctionnaient comme le microcosme d'un espace géographique et cosmique plus vaste qui les rapprochait en particulier des jardins, également orientés à l'époque de la Renaissance en fonction des quatre points cardinaux et de la géographie du territoire $^{49}$. Les guides humanistes sur la manière d'établir une résidence, de Pietro de'Crescenzi à Alberti et Olivier de Serres, s'inspiraient d'ailleurs des conseils des anciens Romains sur la fondation des villes et des camps militaires définis, au cours d'une cérémonie religieuse, sur deux axes nord-sud et est-ouest (cardus et decumanus) et en fonction des vents ${ }^{50}$. Les jardins ou les salles-paysages étaient donc avant tout des microcosmes territoriaux, des images du "lieu" (loci et imagines) inscrits dans un carré et définis par un centre. Michel Foucault applique à ces espaces le terme d' 'hétérotopie" :

"L'hétérotopie a le pouvoir de juxtaposer en un seul lieu réel plusieurs espaces, plusieurs emplacements, qui sont eux-mêmes incompatibles. [...] l'exemple le plus ancien de ces hétérotopies, en forme d'emplacements contradictoires, c'est peutêtre le jardin. [...] Le jardin traditionnel des Persans était un espace sacré qui devait réunir à l'intérieur de son rectangle quatre parties représentant les quatre parties du monde, avec un espace plus sacré encore que les autres qui était comme l'ombilic, le nombril du monde en son milieu (c'est là qu'étaient la vasque et le jet d'eau) [...] Le jardin, c'est la plus petite parcelle du monde puis c'est la totalité du monde"sl.

Les décors des salles-paysages de la Renaissance étaient, eux aussi, pensés sur ce modèle de l'espace universel : l'étendue géographique à l'horizontale, son principe cosmologique à la verticale, sur les parois, la terre, au plafond, le ciel. C'est la traduction littérale, à l'échelle de la salle, de l' "être au monde". Les salles où se développent les décors topographiques à la Renaissance, microcosmes de la ville, du territoire ou du monde, sont en effet presque toujours rattachées à un principe central transcendant : une carte du ciel, comme dans la Sala del Mappamondo à Caprarola ou dans la Sala di Bologna au Vatican, une carte des vents dans la Sala Meridiana à la Tour des Vents au Vatican ou encore la représentation du zodiaque ou des heures comme au château de San Gregorio da Sassola près de Tivoli ou à la Villa Grazioli de Frascati $^{32}$. À la Villa d'Este de Tivoli, la scène d'Hercule au banquet des Dieux sur l'Olympe au plafond du Salone relie l'iconographie du territoire à la figure de son héros tutélaire, mais également à

\section{NOTES}

47. Girolamo Cardano, Opera omnia. 2 vols. Luttich (Ioannus Antonius Huguetan/Marcus Antonius Ravaud) 1663, vol. 2, p. 217 : "Vigilias lætis cogitationibus, studiis, colloquiis, picturarumque amoenarum aspectu compensabis ...". Je dois cette référence à Arnold Witte qui m'a gracieusement permis de lire des passages de son ouvrage de prochaine publication, dans lequel le rapport thérapeutique au paysage dans les Studioli italiens est considéré en détail : Amold Witte, The Artful Hermit : The Palazzetto Farnese as a Counterreformation "Diaeta", L'Erma di Bretschneider, Roma, 2007.

48. Giulio Mancini, Considerazioni sulla pittura (1620), Accademia Nazionale dei Lincei. Fonti e documenti inediti per la storia dell'arte, I, Luigi Salerno (éd.), Roma, 1956-57, vol. I, p. 142. Cité dans Witte, op. cit.

49. Sur l'orientation et la fondation des jardins à la Renaissance, on renverra à la synthèse récente de Malgorzata Szafranska, $o p$. cit., p. 194-198. Sur le symbolisme géographique dans les jardins de la Renaissance, à la Villa Médicis de Castello, à la Villa d'Este de Tivoli, à la Villa Lante de Bagnaia et surtout à la Villa Médicis de Pratolino, voir surtout Hervé Brunon, Pratolino : art des jardins et imaginaire de la nature dans l'Italie de la seconde moitié duXVIe siècle, thèse de doctorat, Université de Paris I PanthéonSorbonne, 2001, 5 vol., chap. 5.

50. Vitruve, De Arch., 1, 6, 1, 7-8, 12-13.

51. Cité dans Monique Mosser, "Hortésie cartographe : de l'écriture classique des parcs français à la calligraphie poétique des jardins anglo-chinois", in Le Paysage des cartes. Genèse d'une codification, actes de colloque (Paris, 1999), Catherine Bousquet Bressolier (dir.), Paris, Musée des Plans-Reliefs, 1999, p. 99 119, p. 104-105. Sur la notion d'hétérotopie associée à l'art des jardins, voir la contribution dans ce numéro de Monique Mosser et Hervé Brunon, "L'enclos comme parcelle et totalité du monde : pour une approche holistique de l'art des jardins".

52. Sur la Sala Bologna, voir Fiorani, op. cit., p. 141-170; Marco Ruffini, Gli affreschi del cardinale Prospero Santacroce nel castello di San Gregorio da Sassola. Rittrato di un committente, Roma, De Luca, 2001. Sur la Villa Grazioli, voir Tantillo Mignosi, op. cit. Pour d'autres exemples de cartes et de globes célestes, voir Jacob Hess, "On some celestial maps and Globes of the Sixteenth Century", Journal of the Warburg and Courtauld Institutes, 30, 1967, p. 406-409. 
l'ancêtre mythique de la famille d'Este ${ }^{53}$. Comme dans d'innombrables cycles topographiques, des miniatures des Mois dans les Très riches Heures $d u$ duc de Berry aux décors de la Villa Giulia à Rome et du Palais Farnèse de Caprarola, l'espace est associé au temps, temps historique et temps cosmique. La représentation des saisons, du calendrier zodiacal, des vents, implique le passage d'une structure microscopique (le lieu) à une structure macroscopique (la Création), dont elle est le miroir.

L'origine de cette formule remonte certainement à l'Antiquité et à la décoration des salles de cérémonie des palais impériaux où l'origine divine du pouvoir temporel des souverains était, pour ainsi dire, matérialisée dans l'espace tridimensionnel de la pièce. Ainsi, les cartes célestes qui décorent les salles de la Renaissance rappellent, à bien des égards, le décor de la salle de banquet principale de la Domus Aurea de Néron, à Rome dont la voûte, comme le raconte Suétone, opérait, comme la voûte céleste, une constante révolution au rythme des heures du jour et de la nuit ${ }^{5 \downarrow}$. On mentionnera aussi les constellations peintes sur les voûtes du Palais de Septime Sévère sur le Palatin ${ }^{5 s}$. Enfin, le célèbre Aviarium ou Ornithon de Varron, avec son dôme céleste décoré d'une rose et des figures des vents, et les étoiles du matin et du soir représentées sur la partie basse de la voûte pour indiquer le passage du temps, constituait également un modèle célèbre connu à la Renaissance ${ }^{56}$.

Le remplacement progressif au XVII e siècle des décors peints à fresque par des tableaux peints sur toile ou par des tapisseries réduira considérablement la capacité de ces salles à construire de si subtils espaces-paysages virtuels. Néanmoins, le rapport entre les tableaux de paysage et le paysage vu depuis la villa continuera, dans de nombreux cas, d'être significatif. Les pendants réalisés par Claude Lorrain pour le Casino Belrespiro de la Villa Pamphilj à Rome vers 1646-1650 furent par exemple conçus en relation étroite avec l'architecture du Casino, l'atmosphère des jardins et les vues sur Rome et la campagne romaine. Comme l'a récemment suggéré Mirka Bene ?. "L'expérience visuelle représentée dans les tableaux faisait écho à la réalité des vues depuis le Casino de la villa et vice-versa", si bien que "les deux paysages de Claude devaient ressembler aux vues depuis les fenêtres de la Villa elle-même, mais merveilleusement re-construites par les règles de l'art" ${ }^{\prime}$.
Claude se plaçait, on l'a vu, au sein d'une tradition longuement établie qui légitime amplement la lecture apparemment audacieuse de Mirka Bene ? En vérité, la méthodologie est simple. Il s'agit de repenser les œuvres d'art non pas comme des totalités fermées, mais comme des détails au sein d'une expérience visuelle plus vaste sur lesquels on s'arrête un instant et qui informent et transforment ce que l'on vient de voir et ce que l'on verra. Daniel Arasse, écrivant sur le détail dans la peinture de paysage classique, avait bien compris que ce va-etvient du regard constituait "une donnée fondamentale du succès de la peinture de paysage" : "...le parcours du regard se constitue d'un va-et-vient du détail à l'ensemble et de l'ensemble au détail [...] l'effet de plaisir du tableau tient à cette possibilité d'oscillation, entre rapprochement et mise à distance de ses parties [...] la dislocation de l'ensemble en une somme (ouverte) de moments successifs et le déplacement des points de vue constituent une modalité essentielle de la réception du paysage en peinture" ${ }^{98}$. Cette modalité du regard qui parcourt l'espace en un jeu d' "oscillation interminable du trop près et du trop loin" s'applique donc tout autant à un espace réel qu'à un espace pictural dans lequel s'investit spatialement un sujet regardant. Une oscillation entre intérieur et extérieur, entre un détail et une vue d'ensemble, allant du particulier au général, qualifie le comportement universel du

\section{NOTES}

53. Sur la représentation d'Hercule dans le Salone en rapport avec l'iconographie du jardin, voir nos remarques dans Ribouillault, «Le Salone... », op. cit., p. 68-70, 79.

54. Suétone, Ner., 6, 2. Voir pour ces décors antiques Courtright, op. cit., p. 100 qui discute leur influence sur la conception de la Tour des Vents de Grégoire XIII au Vatican ainsi que Karl Lehmann, "The dome of Heaven », Art Bulletin, 27, 1945, p. 1-27.

55. Dio Cassius Cocceianus, Hist. Rom., 77. 11. Lehmann, op. cit., p. 8, 24.

56. Varron, De Re Rus., 3, 5, 17 ; Lehmann, op. cit., p. 19-20. 57. Mirka Bene?, « Claude Lorrain's Pendant Landscapes of 1646-1650 for Camillo Pamphilj, Nephew of pope Innocent X. Classicism, Architecture, and Gardens as Contexts for the Artist's Roman Patronage », Storia dell'arte, 112, 2005 , p. 37-60, p. 48.

58. Arasse, op. cit., p. 164. 
Fig. 3. Benedetto et Bonifacio Bembo, Voûte de la Camera peregrina aurea, fresque, vers 1460, Torchiara, Castello dei Rossi.

regard et de la connaissance. À la Villa d'Este à Tivoli, c'est précisément en ces termes qu'Umberto Foglietta décrivait, en 1569 , son expérience de cet espace merveilleux :

"L'aspect de l'œuvre dans son entièreté et la splendeur de ses différentes parties sont tels qu'ils surpassent de beaucoup non seulement les facultés de l'écrivain, mais aussi la puissance de l'imagination. Un seul regard fait parfaitement comprendre comment s'organise la villa, mais cela n'advient pas tout d'un coup, car l'observation individuelle des parties fait grandir peu à peu l'admiration [...]. En fait, le premier regard me saisit l'âme et les yeux d'une stupeur immédiate, et il s'en fallut de peu pour que je n'en perde les sens. Ensuite, me repre- nant et regardant les différentes parties de la villa, je commençai à les admirer plus en détail, et je fus alors véritablement convaincu que ce lieu n'aurait jamais fini de surprendre" $\$ 9$.

\section{Denis Ribouillault}

59. Uberti Folietae, Tyburtinum Hippolyti EstII cardinalis Ferrariensis ad Flavium Ursinum Car. Amplissimum, Tivoli, 1569, trad. it. F. Sciaretta, Tivoli, 2003, p. 2-3. Sur ce passage, voir aussi Ribouillault, "Le Salone", op. cit., p. 84-86. 